\title{
Verification-Guided Soft Error Resilience
}

Sanjit A. Seshia

Wenchao Li

Subhasish Mitra

Electrical Engineering and Computer Sciences University of California at Berkeley

Technical Report No. UCB/EECS-2006-118

http://www.eecs.berkeley.edu/Pubs/TechRpts/2006/EECS-2006-118.html

September 26, 2006 
Copyright @ 2006, by the author(s).

All rights reserved.

Permission to make digital or hard copies of all or part of this work for personal or classroom use is granted without fee provided that copies are not made or distributed for profit or commercial advantage and that copies bear this notice and the full citation on the first page. To copy otherwise, to republish, to post on servers or to redistribute to lists, requires prior specific permission. 


\title{
Verification-Guided Soft Error Resilience
}

\author{
Sanjit A. Seshia \\ Wenchao Li \\ Department of Electrical Engineering and Computer Sciences \\ University of California, Berkeley \\ Berkeley, CA 94720-1770 \\ \{sseshia,wenchao\}@eecs.berkeley.edu \\ Subhasish Mitra \\ Departments of Electrical Engineering and Computer Science \\ Stanford University \\ Stanford, CA 94305 \\ subh@stanford.edu
}

September 26, 2006

\begin{abstract}
Algorithmic techniques for formal verification can be used not just for bug-finding, but also to estimate vulnerability to reliability problems and to reduce overheads of circuit mechanisms for error resilience. We demonstrate this idea of verification-guided error resilience in the context of soft errors in latches. We show how model checking can be used to identify latches in a circuit that must be protected in order that the circuit satisfies a formal specification. Experimental results on a Verilog implementation of the ESA SpaceWire communication protocol indicate that the power overhead of soft error protection can be reduced by a factor of five using our approach, for a fairly comprehensive formal specification.
\end{abstract}

\section{Introduction}

Technology scaling to $65 \mathrm{~nm}$ and below has caused reliability problems to become a dominant design challenge. In fact, design today can be seen as a process of achieving a trade-off between performance, power, and reliability. Problems arise due to soft (transient) errors, aging, environmental and device parameter variations, and aggressive deployment to reduce power and increase performance. In particular, soft errors can be significant contributors to system-level silent data corruption and have been the subject of much recent research $[6,18]$.

There is therefore a pressing need for error-resilient design as well as estimation of the system-level impact of circuit-level errors. For soft errors in latches and flip-flops, there are many protection techniques already available; we point the reader to a recent papers $[19,18]$ for relevant references. However, circuit mechanisms for error resilience come at the price of increased power and area overheads, and possibly reduced performance. As an example, we cite recent fault injection experiments on a microprocessor design [23]. The authors report that protecting $60 \%$ of latches against soft errors sufficed to bring the chip-level soft error rate down to $9 \%$. However, further bringing the error rate down to 0 incurred significant overheads, 


\begin{tabular}{|l|l|l|l|}
\hline Factor & Random Fault Injection & Fault-Free Simulation & Verification-Guided \\
\hline Input coverage & Major concern & Major concern & Exhaustive \\
\hline Error count & Major issue & Non-issue & Exhaustive \\
\hline Applicability & General & Application-specific & General \\
\hline Accuracy & $\begin{array}{l}\text { Needs long } \\
\text { simulation runs }\end{array}$ & $\begin{array}{l}\text { Depends; can be } \\
\text { very pessimistic }\end{array}$ & $\begin{array}{l}\text { Very high, but needs } \\
\text { comprehensive formal spec. }\end{array}$ \\
\hline Scalability & Fair (accuracy can degrade) & Variable & $\begin{array}{l}\text { Problem for large designs } \\
\text { (needs compositional reasoning) }\end{array}$ \\
\hline
\end{tabular}

Table 1: Comparison of verification-guided approach to others

including increasing the power penalty to $18.2 \%$ from $10.6 \%$. There is therefore a need to identify only those circuit resources that must be protected against reliability problems in order for the circuit to meet necessary specifications.

We propose and demonstrate a verification-guided approach to error resilience, wherein algorithmic techniques for formal verification are used to estimate vulnerability to device errors and reduce the overheads of circuit mechanisms for error resilience. The underlying idea is that errors that do not affect circuit correctness, as given by a formal specification, can be ignored. There are several challenges to implementing our approach. First, the formal specification must be exhaustive, capturing the "critical properties" that the circuit must obey under all circumstances. Second, verification techniques must be able to scale to real-world circuits. Third, in order to use formal techniques, we need to create accurate high-level behavioral models of device and circuit errors.

We demonstrate our approach for dealing with soft errors in latches, using the single-event upset (SEU) fault model. Combining a formal SEU model with a formal circuit model, we use the state-of-the-art Cadence SMV model checker [1] to identify latches that must be protected. Our approach is demonstrated by a case study of a publicly available Verilog implementation of end-nodes in the SpaceWire spacecraft communication protocol proposed as a standard by the European Space Agency (ESA) [3]. The results of our experiments show that most latches in the SpaceWire circuit can be left unprotected even for a comprehensive formal specification created from the ESA standards document [11], resulting in a reduction in power overhead from $51 \%$ to just $9 \%$.

Related Work. Our approach can also be viewed as an exhaustive way to perform fault injection of SEUs, guided by a formal specification. Our approach has both pros and cons compared to the alternative approaches of random fault injection $[12,22,15,14]$ and fault-free simulation (e.g., architectural vulnerability factor estimation) [21], and complements them and other techniques [5, 16]. A brief comparison is given in Table 1.

The algorithmic techniques and experiments we propose can be re-used for computing mutation-based coverage metrics for formal verification $[13,7]$. Thus, our approach can be seen as not only meshing with the verification flow, but in fact generating results that can be used both to compute coverage and reduce overheads of error resilience.

\section{Background}

We give some background on the formal verification technique of model checking that is used in this paper. 
Model checking is the most highly automated formal verification technique available today. The technique encompasses algorithmic methods to exhaustively explore all circuit states that are reachable from the initial states of operation. Model checking algorithms form the basis of many recent industry tools for assertionbased verification, sequential equivalence checking, and property checking. Further details may be found in the book by Clarke et al. [10].

Formal specifications can be provided in a variety of ways. For sequential equivalence checking, the specification is captured by a simpler version of the circuit that is considered correct; e.g., a simple, non-pipelined processor can serve as a specification for a complex, pipelined, superscalar version. However, for arbitrary control logic, ASICs, and whole systems, design requirements are often most easily captured as partial specifications, and formalized using temporal logic. Temporal logic forms the basis for the recent IEEE standard 1850 property specification language (PSL) [4].

The experiments reported in this paper use Cadence SMV, a state-of-the-art model checker based on the technique of symbolic model checking [17]. Formal specifications for the main case study were written in linear temporal logic $[20,10]$.

\section{Approach}

We describe our approach in this section, including the overall flow (Section 3.1), underlying formal notions (Section 3.2), and relation to coverage (Section 3.3).

\subsection{Tool Flow}

The overall flow of our approach is depicted in Figure 1. Although we have shown this flow for SEUs, it is also applicable for other classes of circuit reliability problems.

The inputs to the process are the RTL description of the circuit, a formal specification (possibly comprising many properties/assertions), and a formal model of how the circuit reliability problem affects its system-level behavior. We elaborate on the latter point, for SEUs, in Section 3.2. The formal SEU model is automatically compiled into $n$ finite-state machines, each one corresponding to the effect of an SEU on a latch (state variable).

The RTL is abstracted (manually, or otherwise) into a formal model of the circuit. For the work in this paper, this is a description in the input language of a model checker (such as Cadence SMV). However, in general it would depend on the formal verification (FV) tool that is used. Then, for each latch $v_{i}$, the corresponding formal SEU model is composed with the formal circuit model, to obtain the overall input model for the FV tool for $v_{i}$. The overall model is fed to the FV tool along with the formal specification.

A total of $n+1$ runs of the FV tool are performed: one for each of $n$ latches, and a single run to verify that the formal specification holds for the original circuit model in the absence of an SEU. If the overall model for latch $v_{i}$ fails to satisfy the formal specification, we conclude that $v_{i}$ must be protected against an SEU; otherwise, not. Thus, the output of these $n$ runs is a list of latches that must be protected against SEUs.

The RTL is then synthesized, using the list of latches in the process. Power, performance, area, and other circuit parameters are estimated. If the overhead of circuit protection is acceptable, the process stops. Otherwise, the designer must adjust the circuit and/or the specification to meet design objectives. 


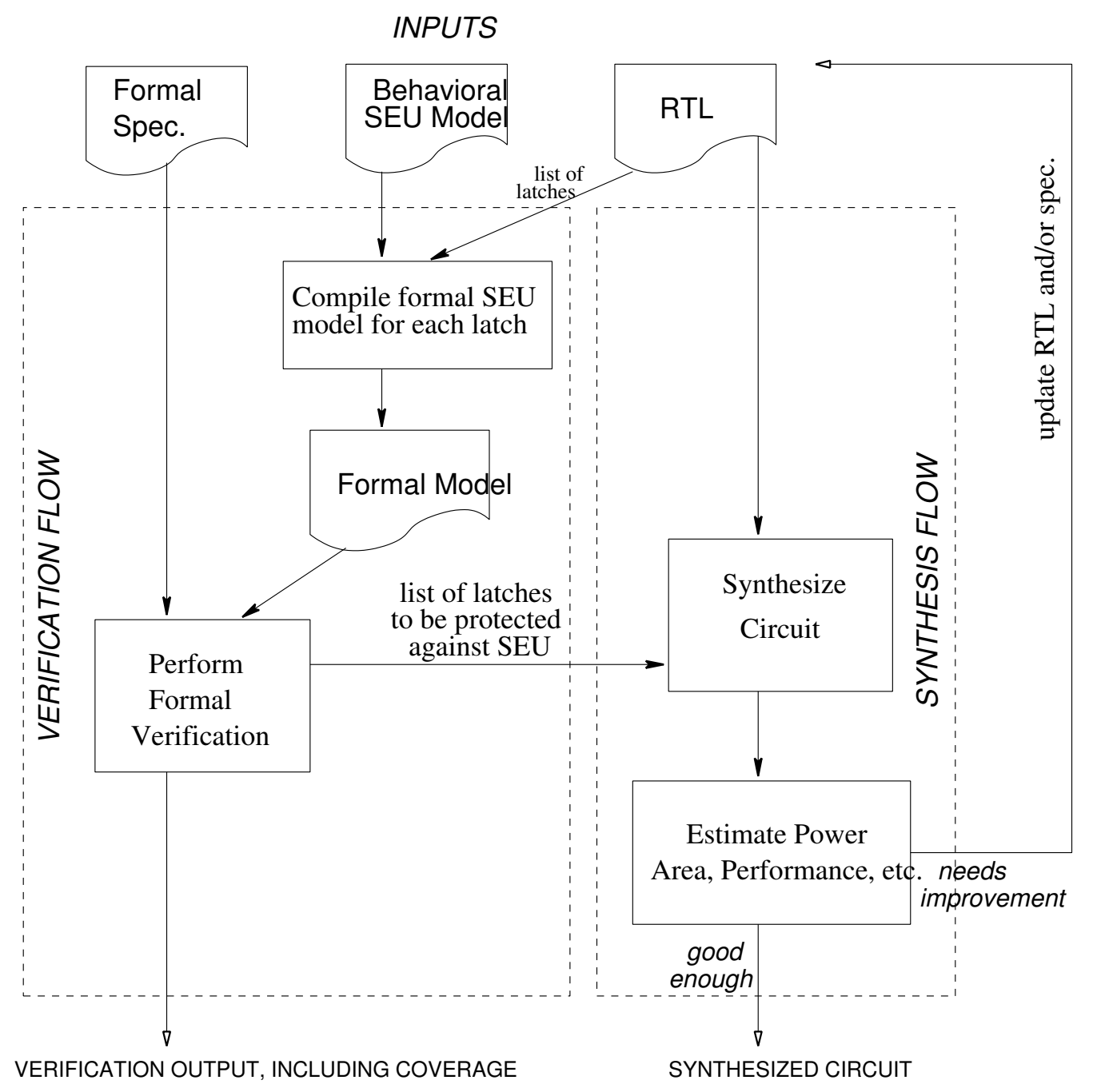

Figure 1: Flow of verification-guided soft error resilience

Note that, although Figure 1 illustrates our approach for SEUs, the flow is independent of the specific reliability problem. In fact, it is also independent of the formal verification technique used. It involves very few changes to existing synthesis and verification flows. In fact, as we note in Section 3.3, it can be viewed as a way to compute mutation-based coverage metrics for verification, so it combines well with existing verification flows.

Note further that the result of this process is highly dependent on the formal specification. A specification that places no constraints on the design will generate results indicating that all latches can be left unprotected! Thus, the results of our approach have a dual use: one of computing the coverage of a formal specification. If all (or almost all) latches can be left unprotected, it indicates that we might need to strengthen the specification. We discuss this point further in Section 3.3.

Finally, the formal specification must cover the critical functionality that the system must maintain even in the face of soft errors. Thus, even when an SEU happens, one can guarantee that the system will only "degrade gracefully." 


\subsection{Formal Model}

As is standard in formal verification, a sequential circuit is formally modeled as a triple $\left(V, \delta, S_{0}\right)$, where $V$ is the set of state variables (latches or state-holding elements) $\left\{v_{1}, v_{2}, \ldots, v_{n}\right\}, \delta$ is the transition relation of the system defining how the system evolves over time, and $S_{0}$ is a Boolean formula over $V$ denoting the set of initial states in which the circuit can begin operation. The transition relation specifies, for each state variable $v_{i}$, how its value in the next cycle $v_{i}^{\prime}$ is obtained. Note that since $\delta$ is a relation, not just a function, non-determinism in the system can be easily modeled.

Given an RTL-level circuit description, such as in Verilog, we manually create a formal model of it as given above. Timing-related details in the RTL are modeled using non-determinism, so that the resulting formal model exhibits a superset of the actual system behaviors. Any verification performed on the formal model will then be conservative.

In the SEU fault model, there is a single bit flip during an arbitrary cycle of circuit operation. Figure 2 shows the formal model of an SEU in latch $v_{i}$.

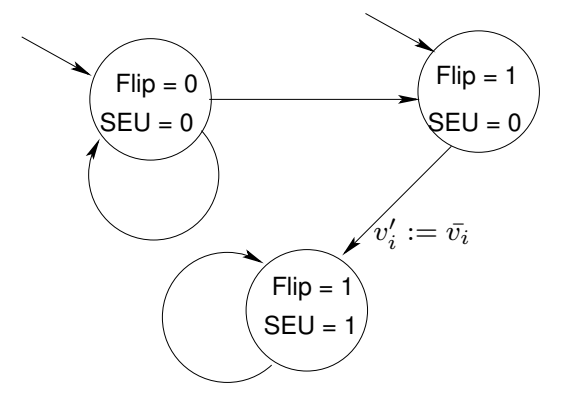

Figure 2: Formal model of impact of an SEU on Boolean state variable $v_{i}$

This model comprises two state bits: Flip and SEU, and has two initial states. Flip is initially either 0 or 1 , if 1 , it indicates that the system will encounter an SEU in the next cycle. SEU records whether an SEU has occurred. Thus, the three-state automaton shown in Figure 2 models an SEU in $v_{i}$. When SEU goes from 0 to 1 , it also flips the value of latch $v_{i}$, as shown by the label on the transition $v_{i}^{\prime}:=\bar{v}_{i}$.

\subsection{Coverage}

Several coverage metrics have been proposed for formal verification [13, 7]. The main ones, some of which are in industrial use, are based on mutating the circuit model and checking whether the specification continues to be satisfied. If yes, then the specification is not exhaustive enough, and the verification engineer must extend it. The intuition is that an exhaustive formal specification should closely characterize the set of correct circuit behaviors.

Consider our formal model of an SEU in latch $v_{i}$, shown in Figure 2. An SEU is a form of mutation, in fact very similar to those considered in the literature $[13,7]$. Thus, if the list of latches to be protected against an SEU (indicated in Figure 1 as an input to synthesis) is empty, it is cause for suspecting the coverage of the specification.

Thus, our approach is not only useful for analyzing the impact of soft errors, but the results can also be 
re-used for computing coverage metrics. In fact, extending our approach beyond soft errors can help define and compute new coverage metrics for formal verification.

\section{Case Studies and Results}

We have performed experiments with standard model checking benchmarks [2] as well as a large case study using third party-written Verilog.

Our results on the model checking benchmarks are only briefly mentioned, as we are unsure how extensive the formal specifications were. A subset of the benchmarks ("eijk" examples) were from sequential equivalence checking, where we were unable to find any latches that could be left unprotected. The reason is that the specification is too restrictive, requiring the outputs to be equal on all cycles. In fact, for such examples, latches could only be left unprotected if the next-state and output behavior of the circuit was independent of them, in which case they could be optimized away.

The rest of this section describes our main case study, an implementation of a node in the SpaceWire network.

\subsection{SpaceWire}

SpaceWire [11] is a network for space applications composed of nodes and routers interconnected through bi-directional high speed data links. According to the SpaceWire website hosted by the ESA, it has been used in missions of the ESA as well as NASA and JAXA.

The SpaceWire standard [11] describes 6 protocol levels - physical, signal, character, exchange, packet, and network. In this paper, we are concerned with the exchange level that defines the protocol for link initialization, flow control, and link error detection and recovery (similar to the more widely known Transmission Control Protocol, TCP). We downloaded a specific Verilog implementation of a SpaceWire end node from opencores.org [3] which was not written by our group. The Verilog was manually translated into the input language for the Cadence SMV model checker. English language specifications from the standards document [11] were translated into formal specifications in linear temporal logic and inserted into the SMV file as assertions to be checked. The Verilog (and the corresponding SMV model) had to be fixed in a few places as a result of our initial experiments to formally verify it. All results discussed below are with respect to this fixed SMV model.

\subsubsection{Overview and Model}

The block diagram of a SpaceWire end node (comprising encoder and decoder) is given in Figure 3. For purposes of reasoning about the exchange layer control protocol, a SpaceWire end node comprises three modules: a transmitter (TX), a receiver (RX), and a state machine that sends control signals to them (FSM). Generating a SMV model from Verilog involved straightforward transliteration for the most part, retaining the control structure, and only abstracting away some data and timing. The end node includes logic for parity error detection and correction on the data (which can be used for communication channel errors as well as SEUs), so it is the control which is left unprotected and is of particular interest to our analysis of SEUs. 


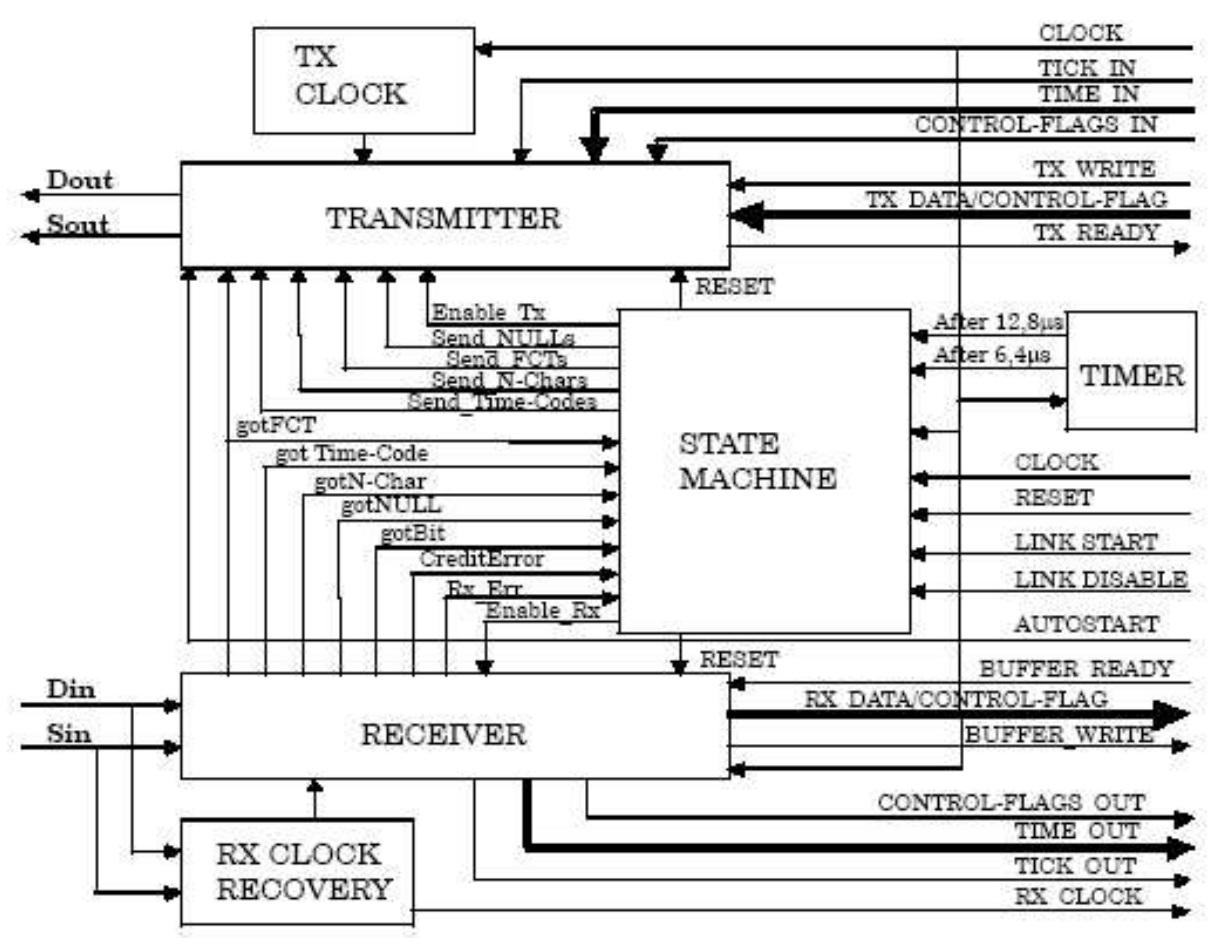

Figure 3: Interfaces within and to a SpaceWire end node. Reproduced from page 57 of [11].

We briefly describe below the operation of the FSM, TX, and RX modules, indicating where state was abstracted away in going to a SMV model. Further details may be found in the standards document [11].

The FSM controls the overall operation of the end node. Its operation is shown in Figure 4. The sequence of ErrorReset, ErrorWait, and Ready provides a mechanism of initializing the SpaceWire node, either coming from a whole system reset or triggered by an error. During this sequence of operation, RX is enabled to receive, but TX is prohibited from sending. In the Started state, TX can send NULL signals to the other end, to establish a connection. Next, the FSM enters the Connecting state where TX is enabled to send flow control tokens (FCTs). When RX receives FCTs, it indicates that the other end has space in its receive buffer for data. The Run state is the state for normal operation where packets flow freely in both directions across the link. The node remains in the Run state until an error occurs or until the link is disabled.

The end nodes communicate over a channel that was modeled in SMV to be capable of dropping or creating parity errors in both control and data packets. (Appropriate "fairness" constraints [10] were imposed on the channel to ensure that a packet would eventually get to its destination, even if dropped several times.)

The transmitter TX is responsible for encoding data (abstracted away in SMV) and transmitting it across the link. Packets are sent according to the following priority, from highest to lowest - Time-Code (system time information), FCT, N-Char (normal characters including data, EOP and EEP) and Null. If there is nothing to send, the transmitter sends NULL to maintain an active link. The transmitter also keeps a credit count of the number of characters that it has been given permission to send. The credit count roughly indicates the space of the opposite receiver buffer.

The receiver RX is responsible for buffering data and passing it on to the host system (abstracted away). It is 


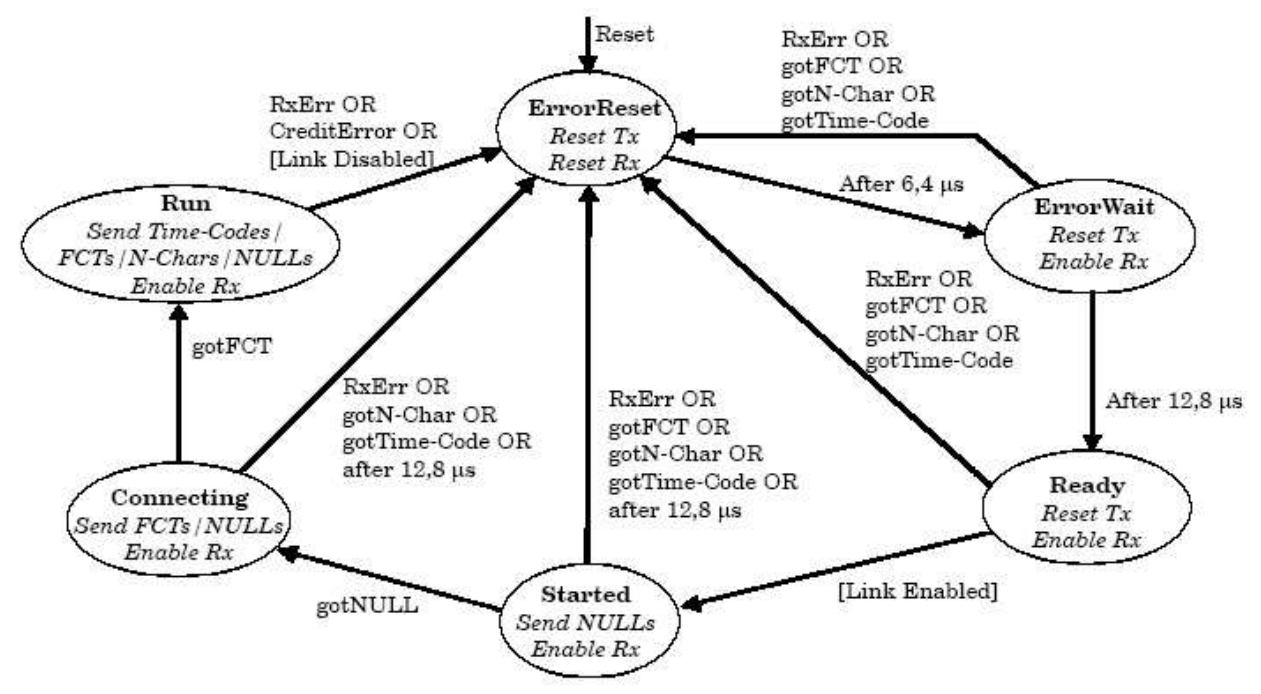

Figure 4: Operation of control finite-state machine in a SpaceWire end node. Reproduced from page 60 of [11].

also responsible for detecting disconnect errors, parity errors, escape errors and credit errors, and reporting these errors to the FSM. When an FCT is received, RX informs TX so that TX can update its credit count accordingly. RX also keeps an outstanding count of the number of characters that it expects to receive.

\subsubsection{Formal Specifications}

We wrote a total of 39 SMV assertions in linear temporal logic, each corresponding to an English-language specification in the standards document [11]. These assertions specify both safety and progress conditions.

Table 2 lists representative assertions. Specifications we wrote fall into five categories, with each category represented in the table. The first set of specifications (row nos. 1 and 2) is on the FSM operation, indicating how and when the system can move between FSM states, as shown in Figure 4. The second is on the interaction between FSM, TX, and RX, exemplified by row 3 in the table that deals with error handling. ${ }^{1}$ The next two sets, exemplified by rows 4 and 5, are on transmitter and receiver operation. The final set of specifications (e.g., row 6) are on the end-to-end communication between two nodes in the SpaceWire network.

Our formal specification is as comprehensive as the corresponding English language specifications in the standards documents. Moreover, note that we have assertions that place safety conditions on the system's behavior as well as state progress conditions indicating that the system is "doing what it should".

\subsubsection{Results}

We created an SMV model 987 lines long (including assertions and fairness constraints) from 1393 lines of Verilog. There are a total of 87 state variables. Some variables, for example, corresponding to counters and

\footnotetext{
${ }^{1}$ Note that row 3 refers to both internal FSM state and the inputs it receives from TX and RX, ending in _i.
} 
FSM state, can generate multiple latches in the synthesized circuit.

Using the formal specifications created from the standards document, we found that all but 10 of these state variables could be left unprotected.

An example of a state variable that must be protected is FSM.state. On a bit flip, this can arbitrarily change the state of the FSM, leading to failure of many assertions, including row 6 in Table 2.

An example of a state variable that need not be protected is FSM.HASgotNULL which is an internal FSM flag that indicates that the end node has received a NULL. This flag is used widely in the FSM logic for error handling, so it was initially somewhat surprising to us that an SEU in it allowed the end node to continue to satisfy all its assertions, including those dealing with errors. It turns out that the error handling logic references not just FSM.HASgotNULL but also another signal RX.gotNULL_o from the receiver, thus providing inherent robustness to SEUs.

Our experiments were performed using a BDD-based version of the Cadence SMV model checker (with dynamic variable reordering turned on). For scalability, all categories of specifications except for the end-toend assertions were verified on a model of a single node communicating with a channel that could generate any message (a conservative check). The total time for our SMV runs for these specifications was 278 seconds with a maximum of 133 seconds for a single run (SMV caches results, thus optimizing overall run-time). The end-to-end assertions were verified on a model comprising two nodes communicating over a lossy channel. This experiment took much longer due to an explosion in the state space -795 minutes with a maximum time of 76 minutes for a single run.

Synopsys Design Compiler was used to generate the final circuit. Latches that did not map to any state variable in Verilog were protected or not based on a structural dependency analysis. Three power consumption numbers were then estimated: for the synthesized circuit without any SEU protection at all, with the BISER protection [23] for all latches, and with BISER protection using our verification-guided classification. The following results were obtained:

\begin{tabular}{lcr} 
Technique & Power(mW) & Overhead \\
\hline No SEU protection & 3.23 & - \\
SEU protection for all latches & 4.85 & $51.0 \%$ \\
Verification-guided SEU protection & 3.52 & $9.1 \%$
\end{tabular}

Thus, using a verification-guided approach one can obtain a $5 \mathrm{X}$ reduction in power overhead of protecting from SEUs using the BISER technique. We believe similar results can be obtained for other SEU protection methods as well, since the fraction of state variables to be protected is small.

\section{Conclusions and Future Work}

We have proposed a verification-guided approach to estimating and reducing the overheads of circuit mechanisms for soft error resilience. Our approach has been demonstrated on a real case study of a third-party Verilog implementation of a component of the ESA SpaceWire network with specifications covering the specified behavior in the standards document [11]. The resulting power savings demonstrate the utility of our approach.

This paper has only taken a first step. Scalability of model checking (and formal verification, in general) is a concern. Encouragingly, we have only used the SMV model checker with its most basic optimiza- 
tions, without either automated abstraction, compositional reasoning, or Boolean satisfiability (SAT) methods $[10,8,9]$. Our approach can also be combined with complementary methods such as random fault injection. Finally, our work has direct connections to the problem of computing coverage metrics for formal verification, which we plan to explore further.

\section{Acknowledgments}

Timothy Loo and Lynn Wang created the initial version of the SpaceWire SMV model. Spencer Chu helped with synthesis and power estimation. This research was supported in part by the MARCO Gigascale Systems Research Center.

\section{References}

[1] Cadence SMV model checker. http://www.kenmcmil.com/ smv.html.

[2] The TIP benchmarks. http://www.cs.chalmers.se//een/Tip/.

[3] SpaceWire Verilog. http://www.opencores.org/projects.cgi/ web/spacewire/overview, July 2005.

[4] IEEE P1850 - standard for PSL - property specification language. http://www.eda.org/ieee-1850/, URL circa Sep.'06.

[5] H. Asadi and M. B. Tahoori. Soft error modeling and protection for sequential elements. In Proc. of the IEEE Intl. Symp. On Defect and Fault Tolerance in VLSI Systems (DFT), pages 463-471, October 2005.

[6] R. C. Baumann. The impact of technology scaling on soft error rate performance and limits to the efficiency of error correction. In Proceedings of the International Electron Devices Meeting, pages 329-332, 2002.

[7] Hana Chockler, Orna Kupferman, and Moshe Y. Vardi. Coverage metrics for formal verification. In Proc. Correct Hardware Design and Verification Methods (CHARME), pages 111-125, 2003.

[8] Edmund M. Clarke, Armin Biere, Richard Raimi, and Yunshan Zhu. Bounded model checking using satisfiability solving. Formal Methods in System Design, 19(1):7-34, 2001.

[9] Edmund M. Clarke, Orna Grumberg, Somesh Jha, Yuan Lu, and Helmut Veith. Counterexampleguided abstraction refinement for symbolic model checking. Journal of the ACM, 50(5):752-794, 2003.

[10] Edmund M. Clarke, Orna Grumberg, and Doron A. Peled. Model Checking. MIT Press, 2000.

[11] European Cooperation for Space Standardization. Space engineering - SpaceWire - links, nodes, routers, and networks (draft ECSS-E-50-12A). http://www.spacewire.esa.int/tech/ spacewire/standards/, November 2002.

[12] K.K. Goswami, R. Iyer, and L.Y. Young. DEPEND: a simulation-based environment for system-level dependability analysis. IEEE Trans. Computers, pages 60-74, Jan. 1997. 
[13] Yatin Vasant Hoskote, Timothy Kam, Pei-Hsin Ho, and Xudong Zhao. Coverage estimation for symbolic model checking. In Design Automation Conference (DAC), pages 300-305, 1999.

[14] M.C. Hsueh, T.K. Tsai, and R.K. Iyer. Fault injection techniques and tools. IEEE Computer, pages 75-82, April 1997.

[15] Ghani A. Kanawati, Nasser A. Kanawati, and Jacob A. Abraham. FERRARI: A flexible software-based fault and error injection system. IEEE Trans. on Computers, 44(2):248-260, 1995.

[16] Smita Krishnaswamy, George F. Viamontes, Igor L. Markov, and John P. Hayes. Accurate reliablity evaluation and enhancement via probabilistic transfer matrices. In Proc. Design Automation and Test in Europe (DATE), pages 282-287, 2005.

[17] Kenneth L. McMillan. Symbolic Model Checking. Kluwer Academic Publishers, 1992.

[18] Subhasish Mitra, Tanay Karnik, Norbert Seifert, and Ming Zhang. Logic soft errors in sub-65nm technologies design and CAD challenges. In Proceedings of the 42nd Design Automation Conference (DAC), pages 2-4. ACM Press, 2005.

[19] M. Nicolaidis. Design for soft error mitigation. IEEE Trans. on Device and Materials Reliability, 5(3):405-418, Sept. 2005.

[20] Amir Pnueli. The temporal logic of programs. In 18th Annual Symposium on Foundations of Computer Science (FOCS), pages 46-57, 1977.

[21] S. S. Mukherjee et al. A systematic methodology to compute the architectural vulnerability factors for a high-performance microprocessor. In Proc. Int'l Symp. Microarchitecture (MICRO), pages 29-40, 2003.

[22] Nicholas J. Wang, Justin Quek, Todd M. Rafacz, and Sanjay J. Patel. Characterizing the effects of transient faults on a high-performance processor pipeline. In Proc. Int'l Conf. Dependable Systems and Networks (DSN), pages 61-70. IEEE Press, 2004.

[23] M. Zhang, S. Mitra, T. M. Mak, N. Seifert, Q. Shi, K.S. Kim, N. Shanbhag, N. Wang, and S.J. Patel. Sequential element design with built-in soft error resilience. IEEE Transactions on VLSI, 2006. 


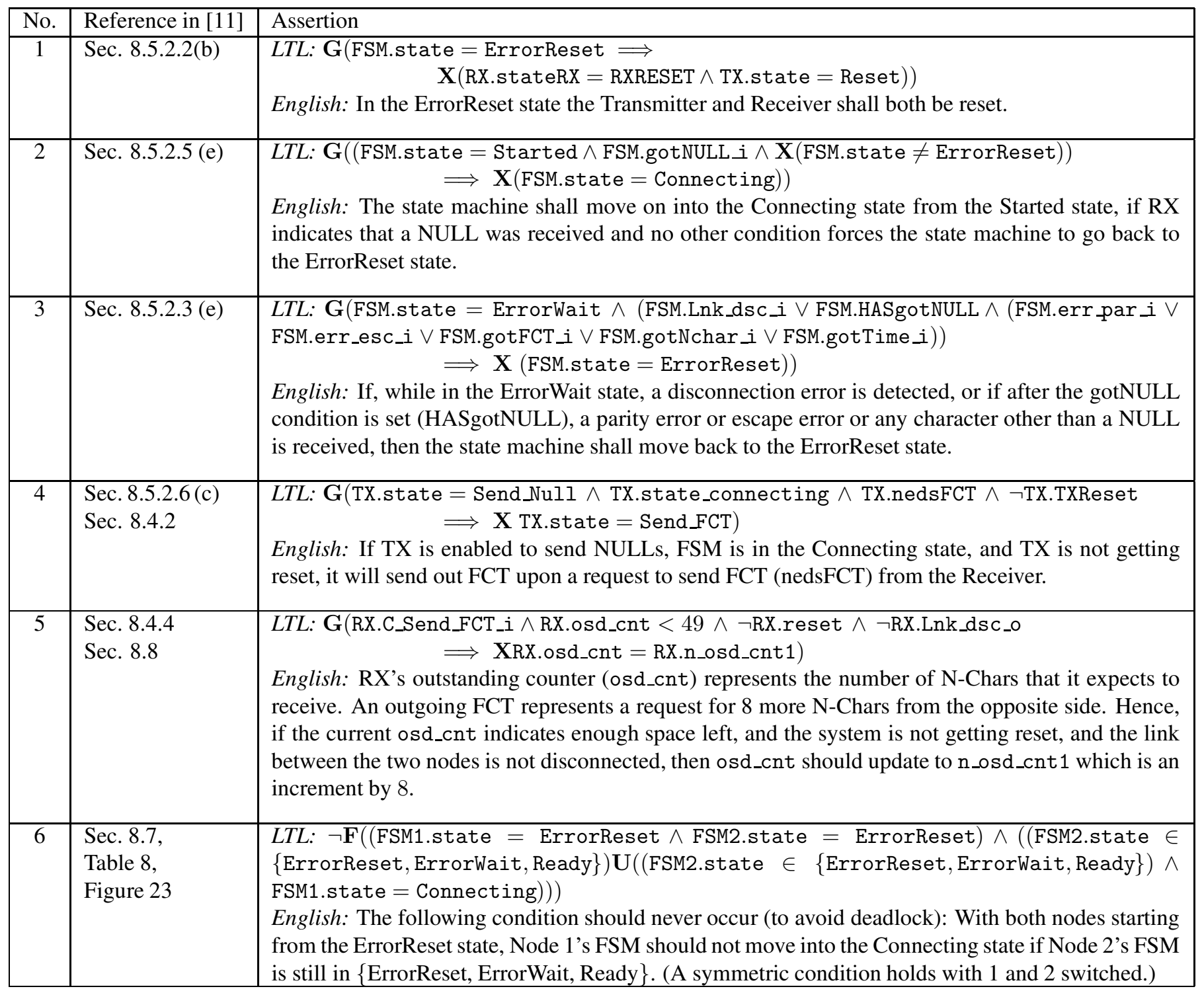

Table 2: Selected formal specifications. $L T L$ indicates a specification in linear temporal logic. 